\title{
How accurate can genetic predictions be?
}

\author{
Jonathan M Dreyfuss ${ }^{1,2^{*}+}$, Daniel Levner ${ }^{3,4 \dagger}$, James E Galagan ${ }^{5,6}$, George M Church ${ }^{3,4}$ \\ and Marco F Ramoni ${ }^{1,7,8^{\wedge}}$
}

\begin{abstract}
Background: Pre-symptomatic prediction of disease and drug response based on genetic testing is a critical component of personalized medicine. Previous work has demonstrated that the predictive capacity of genetic testing is constrained by the heritability and prevalence of the tested trait, although these constraints have only been approximated under the assumption of a normally distributed genetic risk distribution.

Results: Here, we mathematically derive the absolute limits that these factors impose on test accuracy in the absence of any distributional assumptions on risk. We present these limits in terms of the best-case receiveroperating characteristic (ROC) curve, consisting of the best-case test sensitivities and specificities, and the AUC (area under the curve) measure of accuracy. We apply our method to genetic prediction of type 2 diabetes and breast cancer, and we additionally show the best possible accuracy that can be obtained from integrated predictors, which can incorporate non-genetic features.
\end{abstract}

Conclusion: Knowledge of such limits is valuable in understanding the implications of genetic testing even before additional associations are identified.

\section{Background}

Accurate pre-symptomatic prediction of disease and drug response is a vital component of personalized medicine, which could allow for improved clinical decision-making and targeted prevention strategies, easing both the burden and costs of disease [1]. Already, several companies offer consumers personalized risk assessments, lifestyle recommendations, and 'nutraceuticals' based on their genetic profiles [2]. Unfortunately, most genetic factors associated with common traits explain only a small portion of the phenotypic variance (the "missing heritability" problem [3]), making genetic prediction currently difficult [4]. Investment into studies that assay rare variants [5] and the use of informative polymorphisms that do not individually pass stringent statistical tests of association [6] can improve the accuracy of predictions, but the extent to which predictions can be improved is unclear. Thus, identifying the bounds on the accuracy of predictive genetic testing based on

\footnotetext{
* Correspondence: jdreyf@bu.edu

${ }^{\dagger}$ Equal contributors

Deceased

'Partners Center for Personalized Genetic Medicine, Boston, MA 02115, USA

${ }^{2}$ Graduate Program in Bioinformatics, Boston University, Boston, MA 02215,

USA

Full list of author information is available at the end of the article
}

readily-known disease parameters (such as prevalence and heritability) can be an invaluable planning tool.

Although the accuracy of a medical test can be measured in many ways, the concepts of sensitivity and specificity are paramount [7]. Frequently, the test result is continuous (e.g. the individual's predicted risk), while the clinical decision and true outcome are binary (e.g. either the person will get sick or not), so that different thresholds of the test result yield different pairs of sensitivity and specificity. The receiver operator characteristic (ROC) curve depicts this tradeoff between sensitivity and specificity across all possible thresholds, and the area under this curve (AUC) is the most widely used metric to summarize the accuracy of a test. An AUC of 1 indicates perfect prediction while an AUC of 0.5 represents random guessing.

Evidence that a bound on maximum predictive accuracy exists can be found in heritability. The heritability of a trait (in the broad-sense) is the proportion of phenotypic variation in the population that can be attributed to genetic variation; that is, it reflects the contribution of genetic factors relative to environmental ones. Narrowsense heritability measures the corresponding quantity for additive genetic variance only, which excludes genetic effects such as dominance and epistasis. The heritability
C Biomed Central

(C) 2012 Dreyfuss et al.; licensee BioMed Central Ltd. This is an Open Access article distributed under the terms of the Creative Commons Attribution License (http://creativecommons.org/licenses/by/2.0), which permits unrestricted use, distribution, and reproduction in any medium, provided the original work is properly cited. 
of binary phenotypes can be computed directly on the observed binary scale. However, it may also be calculated on a liability scale, where it is assumed that an individual has the binary trait if their risk exceeds a threshold. Both types of heritability can be estimated using family-based studies, such as twin studies [8], and the two scales can be mapped to each other [9].

The impact of heritability on genetic test accuracy can be seen by examining its two extremes: a trait that has $100 \%$ heritability, such as a Mendelian trait, can be predicted with certainty from the genotype; in contrast, a trait with $0 \%$ heritability is not influenced by genetic factors, and thus genetic tests cannot produce any useful information. Previous ground-breaking works have investigated the bounds prevalence and heritability impose on predictive accuracy using simulations [10], analytical results utilizing genotype relative risks and their frequencies [11], and analytical approximations under the assumption of a normally distributed liability $[12,13]$. Here, we mathematically elucidate the absolute bounds on the specificities, sensitivities, and AUC for genetic testing given any values of heritability and prevalence of the tested trait, without making any assumptions about the risk distribution.

\section{Results}

Common complex traits are typically the combined effect of genetic and environmental factors. Since no practical predictor can account for all factors and their interactions, clinical prediction can at best assign probabilistic risks rather than deterministic outcomes. Viewed on the population level, these risk assignments can be seen as comprising a risk distribution, which is an estimate of the population's true risk distribution. Maximal predictive accuracy occurs when the estimated risk matches the true risk.

The prevalence and heritability of any trait restrict the set of possible genetic risk distributions. If we know the risk corresponding to each individual's genetic profile in a large sample, then we can obtain an expression for broad-sense heritability $\left(\mathrm{H}^{2}\right)$ on the binary scale [10]:

$$
\text { heritability }=H^{2}=1-\frac{\sum_{i} \operatorname{risk}_{i}\left(1-\operatorname{risk}_{i}\right)}{\text { risk }(1-r \overline{i s k}) n}
$$

where $i=1, \ldots, n$ indexes people, $n$ is the sample size, risk $_{i}$ is individual $i$ 's genetic risk (i.e. the conditional probability of the trait given genes), and risk is the average genetic risk, which equals the average population risk (see Methods). The meaning of risk depends on the context: for instance, when the phenotype is current disease status, the average risk in the population is its prevalence, whereas in prediction of lifetime illness, risk is the lifetime risk of disease. (When possible, we nonetheless opt for the term prevalence.) Equation 1 mathematically expresses that heritability is the proportion of phenotypic variance explained by the genetic risk distribution.

To mathematically derive the risk distribution that yields the best genetic prediction, we model the distribution as a histogram with equally-spaced bins located from 0 to $100 \%$ representing risk groups, where the height of each bin denotes the proportion of the population who fall into that risk group (for an example, see Figure 1). This approach can define any risk distribution. Indeed, multiple genetic risk distributions can correspond to a given combination of prevalence and heritability; each such distribution, however, can lend itself differently to genetic prediction. Our method is based precisely on determining which such distribution (for a given prevalence and heritability) would allow the best predictive accuracy. Thus, for each combination of prevalence and heritability, we optimized the AUC that would be achieved if everyone's risk were ideally ordered over the set of risk distributions that satisfied the combination of prevalence and heritability; similarly, we maximized the sensitivity for any given specificity, prevalence, and heritability over the set of risk distributions and thresholds that satisfied the constraints.

Using this approach, we have derived the maximum limits on the genetic predictive accuracy of any binary trait given only its prevalence and heritability. These values are tabulated in Additional files 1 and 2 in terms

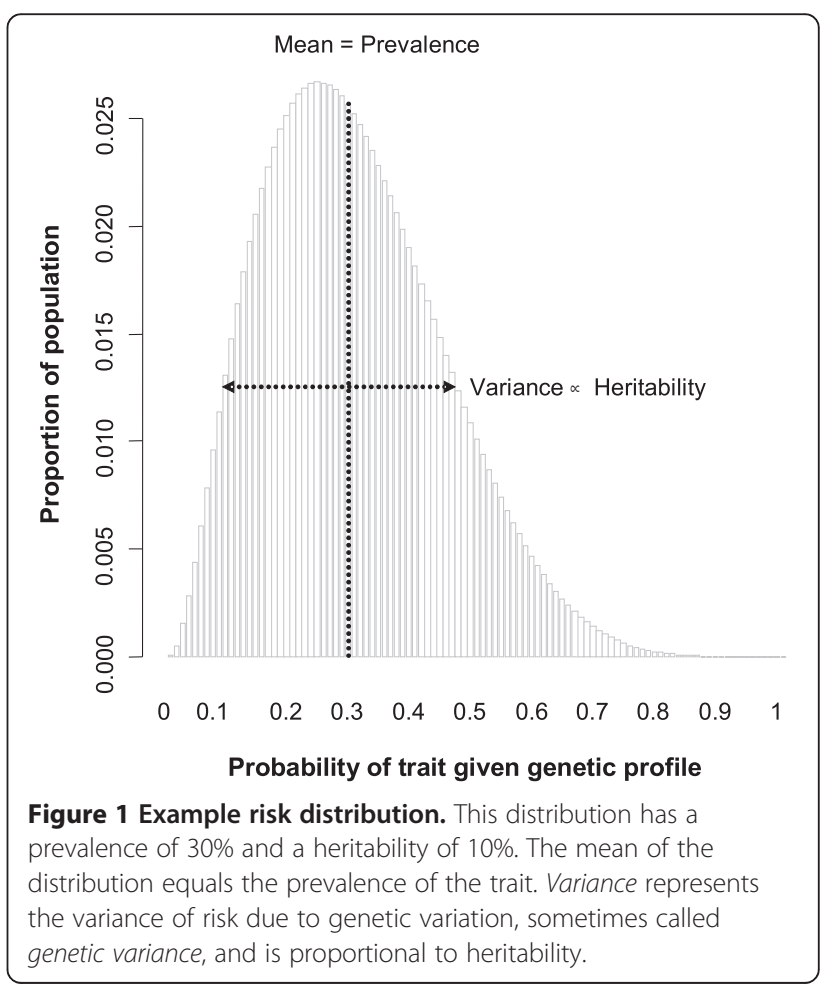


of the AUC and sensitivity/specificity pairs, respectively. Additional file 3 contains computer code in the R software environment [14] for the algorithms we developed. Figure 2 displays AUC limits over all heritabilities for several prevalences, and it includes a comparison with the limits that would exist if genetic risk followed a beta distribution. The beta distribution is a flexible statistical distribution which is consistent with the assumptions of previous analytical approximations of the effect of prevalence and heritability on the ROC curve $[12,13]$, because it can take the shape of countless smooth unimodal risk distributions. Furthermore, unlike previous approximations which deteriorate at high heritabilities [12], the beta distribution limits do not. The limits that the beta distribution imposes on the AUC closely track these previous approximations $[12,13]$ and also match a predictive genomics simulation based on a multiplicative genetic model [10].

Knowledge of this maximal limit on accuracy is beneficial in the case of type 2 diabetes (T2D), where early targeted intervention can be costly but effective [15]. Many prediction studies of T2D have been reported, yet the genetic contribution to their predictive accuracy has been disappointing: genes alone yield $\sim 60 \%$ AUC, and adding genes to clinical risk factors yields incremental improvements of $\sim 1-2 \%$ AUC $[16,17]$. The heritability of T2D per se (as opposed to related continuous traits with higher heritability, e.g. body mass index) was estimated to be $26 \%$ by a population-based twin study [18], with a

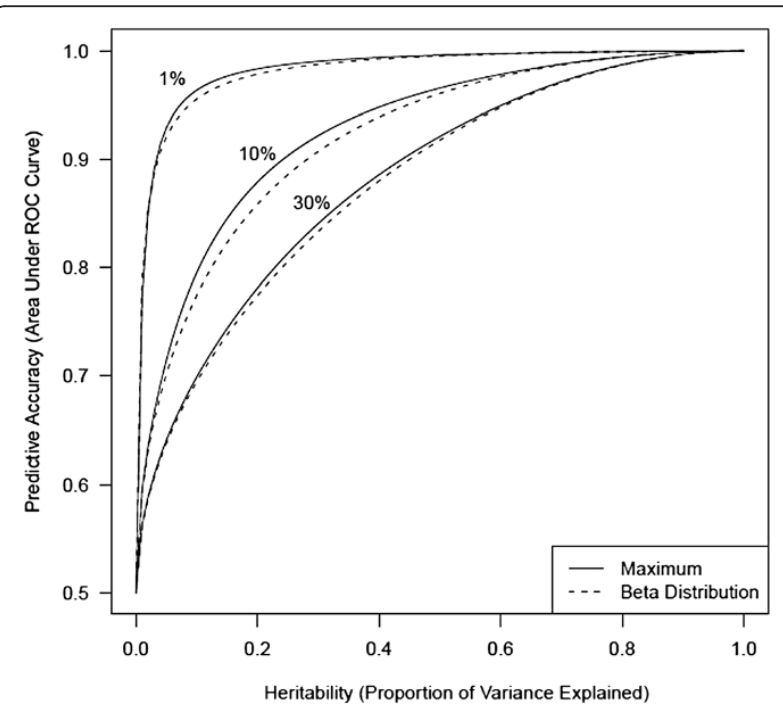

Figure 2 Heritability vs. predictive accuracy. Relationship of heritability (computed on the observed binary scale) or proportion of variance explained to the maximal upper limit on AUC. The numbers next to the curves represent the prevalence. The maximal AUCs are compared with those that would exist if the genetic risk distribution followed a beta distribution, which is consistent with previous reports $[10,12,13]$. prevalence of $13 \%$. Applying our method to these statistics determines the maximum sensitivity/specificity pairs displayed in Figure 3, which show that, for example, if a specificity of $99 \%$ is desired, sensitivity cannot exceed $36 \%$, and that if a sensitivity of $99 \%$ is desired, specificity cannot exceed $74 \%$. Similarly, they determine the maximum achievable AUC for genetic prediction of lifetime T2D to be $89 \%$. This motivates the search for additional genetic factors influencing risk for T2D.

Breast cancer has the same maximal AUC as T2D, albeit with a distinct ROC curve from T2D. Breast cancer was found to have a prevalence of $4 \%$ [19], and we calculated its heritability on the binary scale to be $11 \%$ (see Methods), which yields a maximum AUC of $89 \%$. Although this is the same maximum AUC as for T2D, the sensitivity/specificity pairs for breast cancer (Figure 3) are not identical to those for T2D, owing to the different disease parameters. For example, to reach a specificity of $99 \%$, sensitivity cannot exceed $24 \%$, which is substantially lower than the corresponding maximal sensitivity of T2D when specificity is $99 \%$. The divergence of these two ROC curves as specificity approaches 100\% illustrates the importance of identifying the maximal ROC curve, rather than relying on the maximal AUC alone.

Heritability is the proportion of phenotypic variance explained by all genetic factors, but our analytic approach can treat the proportion of phenotypic variance explained by any particular set of factors. If the proportion of phenotypic variance explained by a particular set of genes is known, that proportion of variance explained could be substituted for heritability in our model. For instance, if a subset of genes could explain $50 \%$ of the genetic variance of T2D (i.e. explain 13\% of phenotypic variance), then the maximum achievable AUC of this subset would be $80 \%$.

Our method can also be applied in elucidating the maximum accuracy of predictors that integrate features such as gene expression, de novo mutation, body mass index, and lifestyle (which are not fully inherited). The proportion of variance explained by such an integrated predictor can then be greater than heritability. When there are no gene-environment interactions, this difference is the proportion of phenotypic variation that these features explain independently of genes. For example, weekly physical activity can explain $4 \%$ of phenotypic variance of T2D (see Methods), is moderately heritable [20], and was found to not interact with well-known gene variants in T2D [21]. Accordingly, the proportion of variance explained by the integrated predictor comprised of genomic profile and physical activity does not increment by the full $4 \%$ beyond the heritability of T2D. If the proportion of $\mathrm{T} 2 \mathrm{D}$ variance that physical activity explains independently of genes was known to be only $3 \%$, say, then the integrated predictor's maximum AUC 


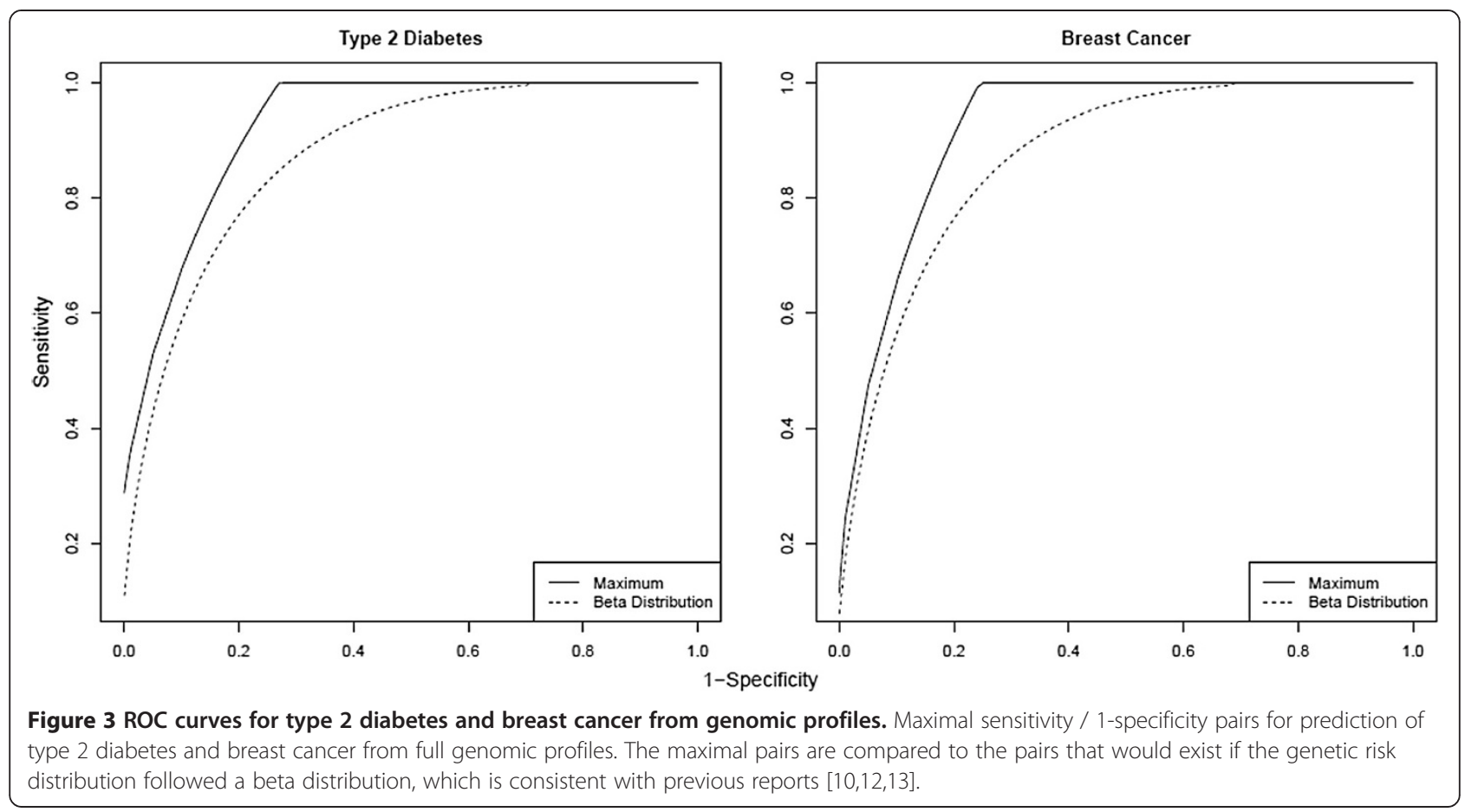

would be calculated based on a proportion of variance explained of $29 \%$ (sum of $26 \%$ and $3 \%$ ), which yields a maximum AUC of $90 \%$. If, however, we did not have an estimate for the proportion of T2D variance that physical activity explains independently of genes, then we could conservatively use $4 \%$ in the previous calculation, yielding a similar AUC. This analysis applies to predictors based on non-genetic features that are supplemented by genetics. In general, the estimation of the proportion of variance explained by integrated predictors is complicated by the interaction of genetic and non-genetic features; our method can nonetheless be applied when the interaction can be estimated or bounded. Note that genetic testing alone can still accurately predict outcome for some small, extreme risk groups (such as those with highly penetrant variants), but such a test will not benefit the general population without both a high sensitivity and specificity [22].

\section{Discussion}

Our results are general and apply to any binary trait, and they rely on only two commonly estimated parameters. Although the quality of the results is only as good as the estimates of prevalence and heritability for the population in question, our method allows for ranges of prevalences and heritabilities to be considered, which can provide important insight into predictive accuracies. Nonetheless, care must be taken when applying these statistics, as different estimates apply in different situations. For example, in assessing limits to the prediction of lifelong risk, lifelong risk estimates should be used in place of prevalence estimates. In particular, the ballooning lifelong risk of T2D in the USA [23] implies genetic prediction of lifetime T2D will become more difficult.

The method that we present here can also be used to determine the potential benefit of a future genomewide association study (GWAS) in improving predictive accuracy. To do so, we refer to estimates of GWAS predictive power that were cleverly derived either by simulation studies [24] or closed-form considerations [25]. Both approaches measure the potential GWAS benefit in terms of the correlation of individuals' genetic risk as predicted by the GWAS to their true genetic risk. We can use our results to connect this measure to AUC and sensitivity/specificity pairs by converting this correlation to a proportion of phenotypic variance explained. If $H^{2}$ is the broad-sense heritability and $r$ is the correlation of true to estimated genetic risk, then the proportion of phenotypic variance that the proposed GWAS may explain, $R^{2}$, is given by [12]:

$$
R^{2}=r^{2} H^{2}
$$

Using this approach, one may evaluate a proposed GWAS based on parameters such as sample size and the number of loci sampled.

Heritability estimates for any binary trait can be used by our method. Broad-sense heritability estimates are needed to cap predictive accuracy, since genetic predictors can exploit dominance and epistatic interactions not measured by narrow-sense heritability 
estimates. However, if a genetic predictor is constructed as an additive model in line with the assumptions of narrowsense heritability, then its maximum accuracy can be calculated using narrow-sense heritability; thus, these estimates can also be used, albeit with a slightly different interpretation. Heritability estimates on the normal liability scale can be used after they are transformed to the observed binary scale, e.g. using the method proposed by Dempster and Lerner [8,9]. Heritability on the binary scale can be sensitive to prevalence [26], but its use avoids the assumption of normally-distributed liability, which requires that the trait be affected by many genes, all with small effect (normally-distributed liability effectively requires a purely unimodal genetic risk distribution). In fact, when variants with particularly large effects do exist-such as $A P O E$ in Alzheimer's disease [27], BRCA1 and BRCA2 in breast and ovarian cancer [28], and LRRK2 in Parkinson's disease [29] — previous authors have suggested simulations in lieu of their analytical approximation [13]. Moreover, because liability cannot be measured, the distributional assumptions on liability are virtually untestable [30].

Our maximal ROC curves (Figure 3) can be substantially higher than those given by the beta distribution, which is an accurate proxy for multiple previous reports $[10,12,13]$, indicating that the maximal accuracies of genetic prediction may be substantially higher than previously thought. This difference highlights the importance that the risk distribution can have in the power of genetic prediction. Furthermore, as we are only now beginning to uncover the risk distributions of common complex diseases, it seems important to understand the absolute, distribution-independent limits on genetic test accuracy, which we present here.

\section{Conclusion}

We have given exact limits on genetic prediction for any binary trait imposed by the epidemiological parameters of prevalence and heritability. Knowledge of these limits can help delineate the maximal benefits associated with genetic testing, which can allow for cost-benefit analyses, regulation, and clinical guidelines regarding genetic testing even before additional associations are identified. We have also illustrated how these limits can help us prioritize the allocation of research resources, by showing how they can assist in the prioritization and design of future association studies. The calculations presented in this paper could further be used to mitigate the possibility of investing in the development of a genetic test which could never be accurate enough to be of clinical relevance.

\section{Methods}

To optimize over the set of risk distributions subject to the disease parameters of average risk and proportion of variance explained (PVE), we modeled a categorical distribution (which resembles a histogram) with $b+1$ bins located at $0,1 / b, 2 / b, \ldots, 1$ representing risk groups, so $i / b$ represents the conditional probability of disease given a set of factors for individuals in risk group $i$ (e.g. people in the $1 / b$ group have risk $1 / b$ ). An example of such a distribution is depicted in Figure 1. The probability that someone falls into bin $i$ is $p_{i}$, where the $p_{i}$ s (for $i=0, \ldots, b)$ sum to one. We restrict the average risk (e.g. prevalence) and PVE (e.g. heritability) using two observations. (1) By the law of total probability, the unconditional probability of disease is simply the mean of the conditional risk distribution, i.e. it is equal to the average risk. (2) The PVE relates to the risk distribution through Equation 1. (Equation 1 can be understood as the $\mathrm{R}^{2}$ from the regression: binary phenotype $=$ risk + error where risk is a probability.)

Now, we perform a brief simplification of Equation 1. Following Wray et al. [24], we denote average risk by $k$, and for generality we work in terms of PVE instead of $\mathrm{H}^{2}$ :

$$
\begin{aligned}
& P V E=1-\frac{\sum_{i} \text { risk }_{i}-\sum_{i} \text { risk }_{i}^{2}}{k(1-k) n} \\
& k(1-k) P V E=k(1-k)-\frac{\sum_{i} \text { risk }_{i}-\sum_{i} \text { risk }_{i}^{2}}{n} \\
& k(1-k) P V E=k(1-k)-k+\frac{\sum_{i} \text { risk }_{i}^{2}}{n} \\
& k(1-k) P V E+k^{2}=\frac{\sum_{i} \text { risk }_{i}^{2}}{n}
\end{aligned}
$$

where $i=1, \ldots, n$ indexes individuals, $n$ is the sample size, and risk $_{i}$ is individual $i$ 's genetic risk. We can relate the right-hand side of Equation 3 to risk groups as follows:

$$
\sum_{i=1}^{n} \operatorname{risk}_{i}^{2} / n=\sum_{j=0}^{b} n_{j} \text { risk }_{j}^{2} / n=\sum_{j=0}^{b} p_{j}\left(\frac{j}{b}\right)^{2}
$$

Here, $n_{j}$ individuals have risk $j / b$, i.e. they are assigned to risk group (histogram bin) $j$, and $p_{j}=n_{j} / n$ is the probability that a random individual is assigned to risk group $j$.

With this model of the risk distribution and constraints, we can identify the best-case AUC and optimal sensitivity/specificity pairs using the procedures detailed below. Because these procedures associate a single genetic risk distribution with the best-case AUC, and a potentially different risk distribution with each optimal sensitivity/specificity pair, it is possible that only some of these sensitivity/specificity pairs may be realizable for a single trait in practice. Consequently, these sensitivity/ specificity pairs cannot be used directly to derive the maximal AUC. 


\section{Area under ROC curve}

To model the AUC, we begin with the random variables $X$ and $Y$ whose probability density functions represent the risk distribution of those who will not and those who will get sick, respectively. These densities can be easily obtained through Bayes rule: $P\left(X=\frac{i}{b}\right)=\frac{(b-i) p_{i}}{b(1-k)}$ and $P\left(Y=\frac{i}{b}\right)=\frac{i p_{i}}{b k}$, where $k$ is the average risk. Then, through its equality to the Mann-Whitney-Wilcoxon U statistic [31], the AUC is equal to $P(X<Y)+P(X=$ $Y) / 2$. We condition on $Y$ to evaluate this expression:

$$
A U C=\sum_{i=1}^{b} P(Y=i / b)\left[\sum_{j=0}^{i-1} P(X=j / b)+\frac{P(X=i / b)}{2}\right] .
$$

We would like to optimize this term, but unfortunately it is not convex, which would undermine our ability to identify the global optimum. However, after we substitute $p_{O}$ with $1-\sum_{i=1}^{b} p_{i}$, our optimization of the AUC becomes a convex optimization problem:

$$
A U C=\frac{\sum_{i=1}^{b} i p_{i}\left[b-\sum_{j=1}^{b} b p_{j}+\sum_{l=1}^{i-1}(b-l) p_{l}+(b-i) p_{i} / 2\right]}{b^{2} k(1-k)}
$$

The numerator of this expression can be conveniently represented as $p^{T} Q p+b^{2} k$, where $Q$ is a symmetric matrix whose entry at row $i$ and column $j$ is $-j(b+i) / 2$ for $i \geq j$.

We then maximize this AUC over the vector $p$ subject to the disease parameters of average risk $(k)$ and proportion of variance explained $(P V E)$ :

$$
\begin{aligned}
& k=\sum_{i=1}^{b}(i / b) p_{i} \\
& k(1-k) P V E+k^{2}=\sum_{i=1}^{b}(i / b)^{2} p_{i}
\end{aligned}
$$

where the sum of the $p_{i}$ 's (for $\left.i=1, \ldots, b\right)$ must not exceed 1 , and each $p_{i}$ is bounded between 0 and 1 .

The parameters $k, P V E$, and $b$ are predefined constants. Note that for $b=100$, as well as for all the values of $b$ we examined, $Q$ is negative definite, so that this is a convex program. Hence, there are efficient solution methods to identify the global maximum. Using the quadprog package [32] in the $\mathrm{R}$ software [14], we solved this program for values of $k$ and $P V E$ with $b=100$. When $b=1000$, all maximal AUCs shown in Figure 2 change by less than $0.01 \%$. In fact, using $b=10$ does not change any of these maximal AUCs more than 1\% from that calculated with $b=1000$. Note also that given an estimated risk distribution vector $p$, a researcher can directly calculate the AUC from the objective function. To calculate the AUC of the beta distribution for given levels of $k$ and $P V E$, we discretized the beta distribution with parameters $a=k(1 / P V E-1)$ and $b=(1-k)(1 / P V E-1)$, which uniquely satisfy the constraints.

\section{Sensitivity/specificity pairs}

To represent each point on the optimal ROC curve, we model the best sensitivity $(\mathrm{Se})$ and specificity $(S p)$ for any given risk threshold $(t / b)$ in terms of the risk distribution. The logic is that the best a genetic test can do is identify true genetic risk, so it will declare those with a risk greater than the threshold as positive and those with a lower risk as negative. Mathematically, the sensitivity of the test is the probability of an individual testing positive for the trait (i.e. having risk of at least $t / b$ ) given that they are truly positive:

$$
\begin{aligned}
& \text { Se }=P(\text { test }+\mid \text { truly }+) \\
& \text { Se }=\frac{P(\text { test }+ \text { \& truly }+)}{P(\text { truly }+)} \\
& \text { Se }=\left(\sum_{i=t}^{b} \frac{i}{b} p_{i}\right) / k \\
& \text { Se }=\frac{1}{b k} \sum_{i=t}^{b} i p_{i}
\end{aligned}
$$

Similarly, we can derive specificity:

$$
S p=\frac{1}{b(1-k)} \sum_{i=0}^{t-1}(b-i) p_{i}
$$

We optimized sensitivity for any given value of specificity, average risk, proportion of variance explained, and threshold using a linear programming model. This was implemented in the lpSolve package in R [14] using 1000 bins. We then optimized the sensitivities over the thresholds to obtain the maximal sensitivity for every specificity, average risk, and proportion of variance explained.

\section{Calculations for examples}

To calculate the proportion of $\mathrm{T} 2 \mathrm{D}$ variance explained by physical activity we used Equation 1, where the risk distribution was defined by the prevalence and the relative risks of exercise [33]. To calculate the heritability of breast cancer on the binary scale we used twice the difference in correlation between monozygotic and dizygotic twin pairs, where correlations were computed on binary outcomes from 44,788 pairs of Nordic twins [34].

\section{Additional files}

Additional file 1: Table of maximum AUCs. These are the maximum AUCs corresponding to Figure 2 for all values of prevalence. Row names represent values of heritability (computed on the observed binary scale) 
or proportion of phenotypic variance explained, and column names represent values of prevalence.

\section{Additional file 2: Table of maximum sensitivities for each} specificity. Rows represent the combination of heritability ( $\mathrm{H} . \mathrm{sq}$ computed on the observed binary scale) and prevalence (Prev), while columns represent specificities. The elements are the maximal sensitivity in each case.

Additional file 3: Archive containing instructions (readme.txt) and computer code (maxAcc.r) to implement our algorithms. The code is written in the free statistical language and environment R (http://www.rproject.org), relies on free $\mathrm{R}$ optimization packages, and is copyrighted by the permissive MIT license (http://www.opensource.org/licenses/mitlicense.html). Updated versions are freely available for download at: http://code.google.com/p/max-accuracy-genetic-pred/.

\section{Abbreviations}

ROC: receiver-operating characteristic; AUC: area under ROC curve; T2D: type 2 diabetes; GWAS: genomewide association study; PVE: proportion of variance explained.

\section{Competing interests}

GMC has advisory roles in and research sponsorships from several companies involved in genome sequencing technology and personal genomics (see http://arep.med.harvard.edu/gmc/tech.html).

\section{Author's contribution}

JMD designed the study, carried out the analysis, and drafted the manuscript. DL designed the study and drafted the manuscript. JEG provided computing resources and helped direct the study. GMC helped direct the study. MFR designed the study and critically revised the manuscript. All authors read and approved the manuscript.

\section{Acknowledgements}

We dedicate this to Marco Ramoni, who tragically passed away in June 2010

\section{Author details}

'Partners Center for Personalized Genetic Medicine, Boston, MA 02115, USA. ${ }^{2}$ Graduate Program in Bioinformatics, Boston University, Boston, MA 02215, USA. ${ }^{3}$ Department of Genetics, Harvard Medical School, Boston MA02115, USA. ${ }^{4}$ Wyss Institute for Biologically Inspired Engineering, Harvard University, Boston, MA 02115, USA. ${ }^{5}$ Broad Institute of MIT and Harvard, Cambridge, MA 02142, USA. ${ }^{6}$ Department of Biomedical Engineering, Boston University, Boston, MA 02215, USA. ${ }^{7}$ Harvard-MIT Division of Health Sciences and Technology, Boston, MA 02139, USA. ${ }^{8}$ Children's Hospital Informatics Program, Boston, MA 02115, USA.

\section{Received: 4 November 2011 Accepted: 1 July 2012} Published: 24 July 2012

\section{References}

1. Collins FS, Green ED, Guttmacher AE, Guyer MS: A vision for the future of genomics research. Nature 2003, 422(6934):835-847.

2. Janssens AC, Gwinn M, Bradley LA, Oostra BA, van Duijn CM, Khoury MJ: A critical appraisal of the scientific basis of commercial genomic profiles used to assess health risks and personalize health interventions. Am J Hum Genet 2008, 82(3):593-599.

3. Manolio TA, Collins FS, Cox NJ, Goldstein DB, Hindorff LA, Hunter DJ, McCarthy MI, Ramos EM, Cardon LR, Chakravarti A, Cho JH, Guttmacher $A E$, Kong A, Kruglyak L, Mardis E, Rotimi CN, Slatkin M, Valle D, Whittemore AS, Boennke M, Clark AG, Eichler EE, Gibson G, Haines JL, Mackay TF, McCarroll SA, Visscher PM: Finding the missing heritability of complex diseases. Nature 2009, 461(7265):747-753.

4. Kraft P, Hunter D: Genetic Risk Prediction - Are We There Yet? N Engl J Med 2009, 360(17):1701-1703.

5. Goldstein DB: Common genetic variation and human traits. N Engl J Med 2009, 360(17):1696-1698.

6. Yang J, Benyamin B, McEvoy BP, Gordon S, Henders AK, Nyholt DR, Madden PA, Heath AC, Martin NG, Montgomery GW, Goddard ME, Visscher PM: Common SNPs explain a large proportion of the heritability for human height. Nat Genet 2010, 42(7):565-569.
7. Pepe MS: The statistical evaluation of medical tests for classification and prediction. Oxford: Oxford University Press; 2003.

8. Visscher PM, Hill WG, Wray NR: Heritability in the genomics era-concepts and misconceptions. Nat Rev Genet 2008, 9(4):255-266.

9. Dempster ER, Lerner IM: Heritability of Threshold Characters. Genetics $1950,35(2): 212-236$

10. Janssens AC, Aulchenko YS, Elefante S, Borsboom GJ, Steyerberg EW, van Duijn CM: Predictive testing for complex diseases using multiple genes: fact or fiction? Genet Med 2006, 8(7):395-400.

11. Moonesinghe R, Liu T, Khoury MJ: Evaluation of the discriminative accuracy of genomic profiling in the prediction of common complex diseases. Eur J Hum Genet 2010, 18(4):485-489.

12. Wray NR, Yang J, Goddard ME, Visscher PM: The genetic interpretation of area under the ROC curve in genomic profiling. PLoS Genet 2010, 6(2): e1000864.

13. So $H C$, Sham $P C$ : A unifying framework for evaluating the predictive power of genetic variants based on the level of heritability explained. PLoS Genet 2010, 6(12):e1001230.

14. Team RDC: R: A language and environment for statistical computing. Vienna: R Foundation for Statistical Computing; 2008

15. Tuomilehto J, Lindström J, Eriksson JG, Valle TT, Hämäläinen $H$, llanneParikka P, Keinänen-Kiukaanniemi S, Laakso M, Louheranta A, Rastas M, Salminen V, Uusitupa M: Prevention of type 2 diabetes mellitus by changes in lifestyle among subjects with impaired glucose tolerance. $N$ Engl J Med 2001, 344(18):1343-1350.

16. Janssens AC, van Duijn CM: Genome-based prediction of common diseases: advances and prospects. Hum Mol Genet 2008, 17(R2):R166-173.

17. Talmud PJ, Hingorani AD, Cooper JA, Marmot MG, Brunner EJ, Kumari $M$, Kivimaki M, Humphries SE: Utility of genetic and non-genetic risk factors in prediction of type 2 diabetes: Whitehall II prospective cohort study. BMJ 2010, 340:b4838.

18. Poulsen P, Kyvik KO, Vaag A, Beck-Nielsen H: Heritability of type II (noninsulin-dependent) diabetes mellitus and abnormal glucose tolerance-a population-based twin study. Diabetologia 1999, 42(2):139-145.

19. Risch N: The genetic epidemiology of cancer: interpreting family and twin studies and their implications for molecular genetic approaches. Cancer Epidemiol Biomarkers Prev 2001, 10(7):733-741.

20. Lauderdale DS, Fabsitz R, Meyer JM, Sholinsky P, Ramakrishnan V, Goldberg J: Familial determinants of moderate and intense physical activity: a twin study. Med Sci Sports Exerc 1997, 29(8):1062-1068.

21. Scott RA, Chu AY, Grarup N, Manning AK, Hivert MF, Shungin D, Tonjes A, Yesupriya A, Barnes D, Bouatia-Naji N, Glazer NL, Jackson AU, Kutalik Z, Lagou V, Marek D, Rasmussen-Torvik LJ, Stringham HM, Tanaka T, Aadahl M, Arking DE, Bergmann S, Boerwinkle E, Bonnycastle LL, Bornstein SR, Brunner E, Bumpstead SJ, Brage S, Carlson OD, Chen H, Chen YD, et al: No Interactions Between Previously Associated 2-Hour Glucose Gene Variants and Physical Activity or BMI on 2-Hour Glucose Levels. Diabetes 2012, 61(5):1291-1296.

22. Janssens AC, Pardo MC, Steyerberg EW, van Duijn CM: Revisiting the clinical validity of multiplex genetic testing in complex diseases. Am J Hum Genet 2004, 74(3):585-588. author reply 588-589.

23. Narayan KM, Boyle JP, Thompson TJ, Sorensen SW, Williamson DF: Lifetime risk for diabetes mellitus in the United States. JAMA 2003, 290(14):1884-1890.

24. Wray NR, Goddard ME, Visscher PM: Prediction of individual genetic risk of complex disease. Curr Opin Genet Dev 2008, 18(3):257-263.

25. Daetwyler HD, Villanueva B, Woolliams JA: Accuracy of predicting the genetic risk of disease using a genome-wide approach. PLOS One 2008, 3(10):e3395.

26. Robertson A, Lerner IM: The Heritability of All-or-None Traits: Viability of Poultry. Genetics 1949, 34(4):395-411.

27. Farrer LA, Cupples LA, Haines JL, Hyman B, Kukull WA, Mayeux R, Myers $\mathrm{RH}$, Pericak-Vance MA, Risch N, van Duijn CM: Effects of age, sex, and ethnicity on the association between apolipoprotein $E$ genotype and Alzheimer disease. A meta-analysis. APOE and Alzheimer Disease Meta Analysis Consortium. JAMA 1997, 278(16):1349-1356.

28. Fu R, Harris EL, Helfand M, Nelson HD: Estimating risk of breast cancer in carriers of BRCA1 and BRCA2 mutations: a meta-analytic approach. Stat Med 2007, 26(8):1775-1787.

29. Healy DG, Falchi M, O'Sullivan SS, Bonifati V, Durr A, Bressman S, Brice A, Aasly J, Zabetian CP, Goldwurm S, Ferreira JJ, Tolosa E, Kay DM, Klein C, Williams DR, Marras C, Lang AE, Wszolek ZK, Berciano J, Schapira AH, Lynch 
T, Bhatia KP, Gasser T, Lees AJ, Wood NW, Consortium IL: Phenotype, genotype, and worldwide genetic penetrance of LRRK2-associated Parkinson's disease: a case-control study. Lancet Neurol 2008, 7(7):583-590.

30. Falconer DS: Introduction to Quantitative Genetics. 2nd edition. New York: Longman; 1981.

31. Hanley JA, MCNeil BJ: The meaning and use of the area under a receiver operating characteristic (ROC) curve. Radiology 1982, 143(1):29-36.

32. Goldfarb $D$, Idnani A: A numerically stable dual method for solving strictly convex quadratic programs. Math Program 1983, 27(1):33.

33. Hu FB, Sigal RJ, Rich-Edwards JW, Colditz GA, Solomon CG, Willett WC, Speizer FE, Manson JE: Walking compared with vigorous physical activity and risk of type 2 diabetes in women: a prospective study. JAMA 1999, 282(15):1433-1439.

34. Lichtenstein P, Holm NV, Verkasalo PK, lliadou A, Kaprio J, Koskenvuo M, Pukkala E, Skytthe A, Hemminki K: Environmental and heritable factors in the causation of cancer-analyses of cohorts of twins from Sweden, Denmark, and Finland. New Engl J Med 2000, 343(2):78-85.

doi:10.1186/1471-2164-13-340

Cite this article as: Dreyfuss et al:: How accurate can genetic predictions be?. BMC Genomics 2012 13:340.

\section{Submit your next manuscript to BioMed Central and take full advantage of:}

- Convenient online submission

- Thorough peer review

- No space constraints or color figure charges

- Immediate publication on acceptance

- Inclusion in PubMed, CAS, Scopus and Google Scholar

- Research which is freely available for redistribution 